\title{
Do high impact exercises produce higher tibial strains than running?
}

Charles Milgrom, Aharon Finestone, Yael Levi, Ariel Simkin, Ingrid Ekenman, Stephen Mendelson, Michael Millgram, Meir Nyska, Nissim Benjuya, David Burr

\begin{abstract}
Background-Bone must have sufficient strength to withstand both instantaneous forces and lower repetitive forces. Repetitive loading, especially when bone strain and/or strain rates are high, can create microdamage and result in stress fracture Aim-To measure in vivo strains and strain rates in human tibia during high impact and moderate impact exercises. Methods-Three strain gauged bone staples were mounted percutaneously in a rosette pattern in the mid diaphysis of the medial tibia in six normal subjects, and in vivo tibial strains were measured during running at $17 \mathrm{~km} / \mathrm{h}$ and drop jumping from heights of 26, 39, and $52 \mathrm{~cm}$.
\end{abstract}

Results-Complete data for all three drop jumps were obtained for four of the six subjects. No statistically significant differences were found in compression, tension, or shear strains with increasing drop jump height, but, at the $52 \mathrm{~cm}$ height, shear strain rate was reduced by one third ( $p=$ $0.03)$. No relation was found between peak compression strain and calculated drop jump energy, indicating that subjects were able to dissipate part of the potential energy of successively higher drop jumps by increasing the range of motion of their knee and ankle joints and not transmitting the energy to their tibia. No statistically significant differences were found between the principal strains during running and drop jumping from $52 \mathrm{~cm}$, but compression $(p=0.01)$ and tension $(p=0.004)$ strain rates were significantly higher during running.

Conclusions-High impact exercises, as represented by drop jumping in this experiment, do not cause higher tibial strains and strain rates than running and therefore are unlikely to place an athlete who is accustomed to fast running at higher risk for bone fatigue.

(Br f Sports Med 2000;34:195-199)

Keywords: bone; strain; biomechanics; stress fractures; impact

Bone is primarily a mechanical organ. It must have sufficient strength to withstand both high instantaneous forces and lower repetitive forces. These forces are a function of both dynamic muscle action and gravitational forces, ${ }^{1}$ which produce strains in the bone that are only $20-30 \%$ of its yield strength of 10000 microstrains. ${ }^{2}$ Repetitive loading, however, can create microdamage that will cause bone to fail at strains that are within the normal physiological range. ${ }^{3}$

Because of the nature of their training, sportspeople and military recruits are at high risk of accumulating bone microdamage that can result in stress fracture. ${ }^{4}$ This usually occurs when they begin new training regimens or increase the intensity of their training. When designing training programmes for athletes and military recruits, it is important to consider the bone strain and strain rates that specific exercises may produce. Exercises that generate high strain and strain rates increase the risk of bone fatigue and stress fracture and should be avoided if not essential to the training goals.

We hypothesised that high impact exercises such as jumping may produce appreciably higher strain and strain rates than more moderate impact exercises such as running. To study this hypothesis, we measured in vivo the tibial strains and strain rates in six subjects during jumps on to a force plate from three heights and during running.

\section{Materials and methods}

Six subjects from the research staff, four male and two female, aged 27 to 52 years, volunteered to be subjects for in vivo tibial strain measurements. All subjects received explanations of the goals, risks, and benefits of their participation in the experiment and signed informed consent forms. The experimental protocol was approved by the human rights committee of the Hadassah University Hospital, Jerusalem, Israel and the Ministry of Health of the State of Israel. All subjects were healthy, with no prior history of medical problems.

IN VIVO STRAIN MEASUREMENTS

Strain gauged staples made from $16 \times 15 \mathrm{~mm}$ bone staples (3M Health Care, St Paul, Minnesota, USA), with a MicroMeasurements EA-06-031DE-350 strain gauge (Measurements Group Inc, Raleigh, North Carolina, USA) bonded to the undersurface of the staple, ${ }^{5}{ }^{6}$ were used to measure in vivo tibial bone strains and strain rates during drop jumps. Three strain gauged staples were inserted percutaneously in a $30^{\circ}$ rosette pattern in the medial aspect of the mid tibial diaphysis.

SURGICAL PROTOCOL

Surgical implantation of the strain gauged staples was performed on an outpatient basis at the Hadassah University Hospital. Surgical implantation was performed in the morning, and the staples were removed on the same day 


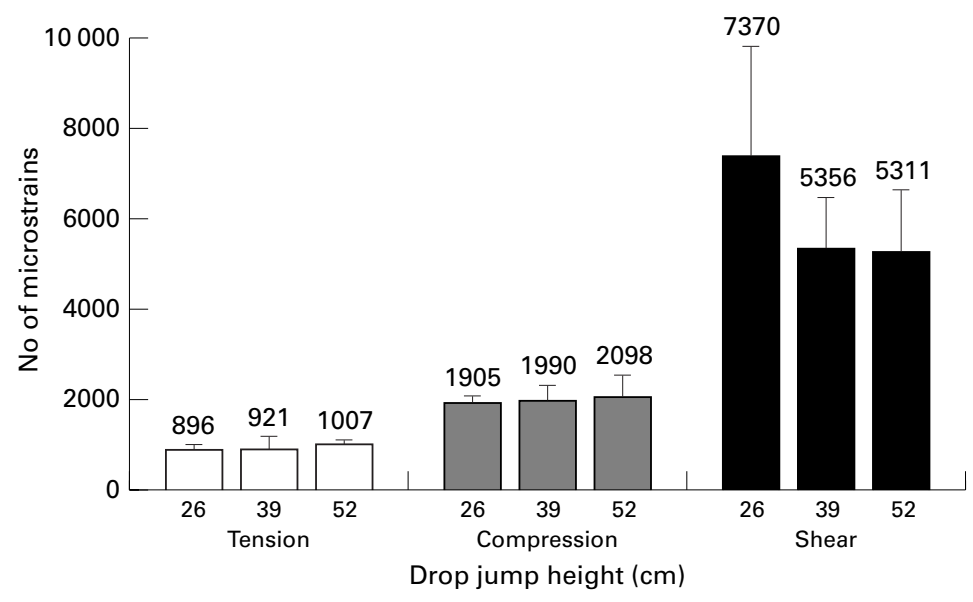

Figure 1 Mean principal tibial strains during drop jumps from different heights. Error bars indicate SEM.

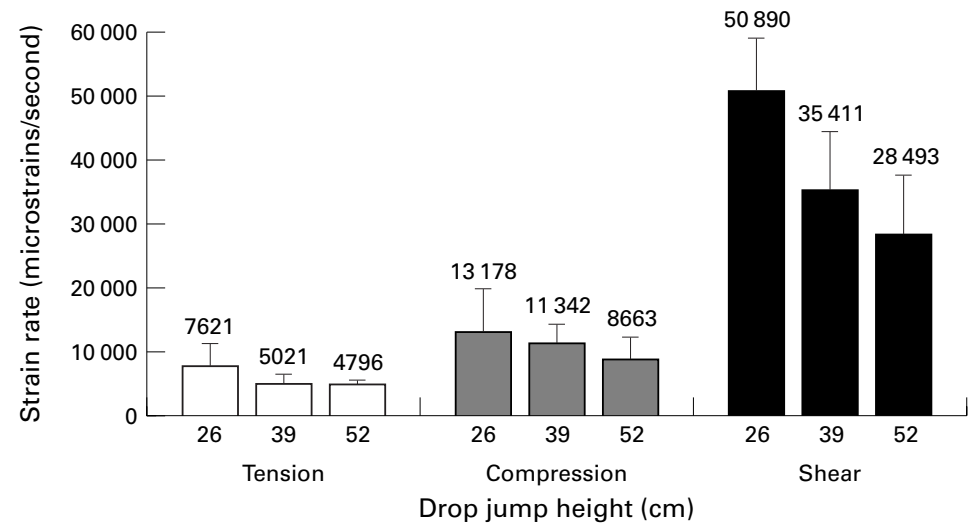

Figure 2 Mean principal tibial strain rates during drop jumps from different heights. Error bars indicate SEM.

after completion of the data collection. Prophylactic intravenous cefonicide was given before each operation.

The right leg was prepared and draped at the level of the mid shaft tibia to facilitate placement of the strain gauged staples in the medial aspect of the tibia. The exact level of the middle diaphysis and the midpoint of the flat medial surface of the tibia were determined. A staple inserter alignment block was then placed centred at this point and the site of the six entrance holes for three staples marked. Surgical anaesthesia was achieved at these six points by local injection of $2.5 \mathrm{ml} 1 \%$ lidocaine and

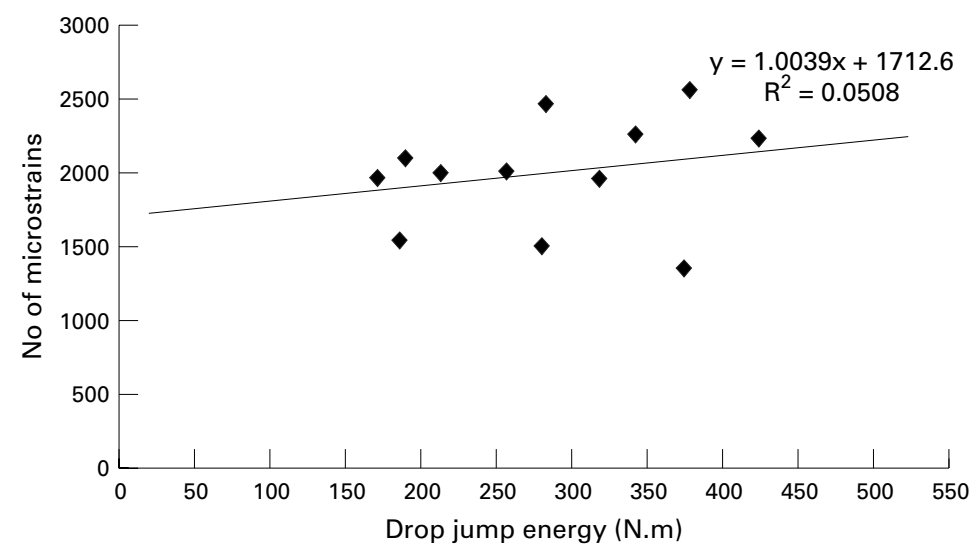

Figure 3 Drop jump energy versus compression strains in tibia.
$2.5 \mathrm{ml} 0.25 \%$ marcaine to the skin, subcutaneous tissue, and the periosteum of the tibia. Surgical stab wounds were made in the skin and subcutaneous tissue at each of the points, and, using the alignment jig and a depth limiting device, the six staple leg entrance sites were drilled $4 \mathrm{~mm}$ into the tibial cortex with a 1.2 $\mathrm{mm}$ drill. The three staples were inserted into the tibia using a specially made inserterimpacter which allows the staple to be driven into the predrilled hole to a depth of $4 \mathrm{~mm}$ within the cortex. A gauze dressing was placed loosely over the staples.

\section{STRAIN GAUGE MEASUREMENTS}

Each staple strain gauge was wired as a $1 / 4$ Wheatstone bridge. The strain gauge exiting wires were connected to a customised portable four channel amplifier. The conditioned amplifier signals were recorded on an FM analogue cassette recorder (HR10; TEAC Corporation, Tokyo, Japan). The amplifier and cassette recorder were carried in a standard Israeli Army multipouch backpack which together weighed $4 \mathrm{~kg}$. Playback was by a separate unit (TEAC MR40) connected to a PC and digitised at $400 \mathrm{~Hz}$.

In vivo tibial strain measurements were made while subjects were running $100 \mathrm{~m}$ on a cinder track at a rate of $17 \mathrm{~km} / \mathrm{h}$ set by a pacer. Drop jump measurements were made sequentially during jumping from wooden blocks of 26,39 , and $52 \mathrm{~cm}$ heights on to a force plate (model OR6-6; Advanced Medical Technology Inc, Watertown, Massachusetts, USA) during which simultaneous in vivo strain measurements were made. Subjects did not practice the technique of jumping from blocks on to a force plate before the actual recordings. The technique was described to them as consisting of a small horizontal jump, just enough to clear the blocks, followed by a vertical drop on to the force plate. The technique of drop jumping was demonstrated by a member of the research team. The jumps were video recorded for determination of knee and ankle range of motion during the drop jumps. In addition, subjects performed a standard walk at $5 \mathrm{~km} / \mathrm{h}$ before and after the run and before and after the drop jumps to check the reproducibility of recordings. All subjects wore Nike Air Max shoes for the experiment.

DATA ANALYSIS

Raw data from the three strain gauged staples were processed using a custom written computer program running under Windows, which takes the digitised amplified signal outputs of the three gauges, filters them at $5 \mathrm{~Hz}$, performs baseline corrections, and derives the maximum principal compression and tension strains and their axes, the maximum engineering shear strains, and the strain rates.

For drop jumps the major outcome variables were the maximum principal strains and strain rates when drop jumping was performed from different heights. The statistical analysis tests whether the mean of each of the major outcomes differs for the drop jump heights using block analysis of variance. The drop 


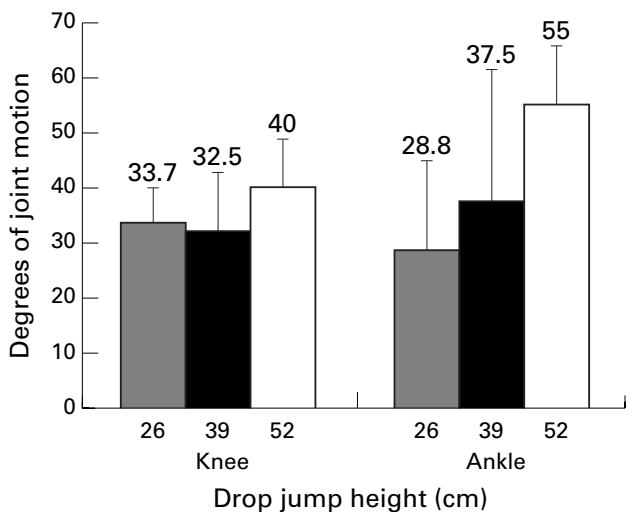

Figure 4 Range of motion of knee and ankle joints versus drop jump height. Values are mean (SEM).

jump energy for each subject at each height was calculated by the formula $\mathrm{E}=\mathrm{mgh}(\mathrm{E}=$ energy, $\mathrm{m}=$ mass, $\mathrm{g}=$ force of gravity, $\mathrm{h}=$ height of drop jump block). The calculated drop jump

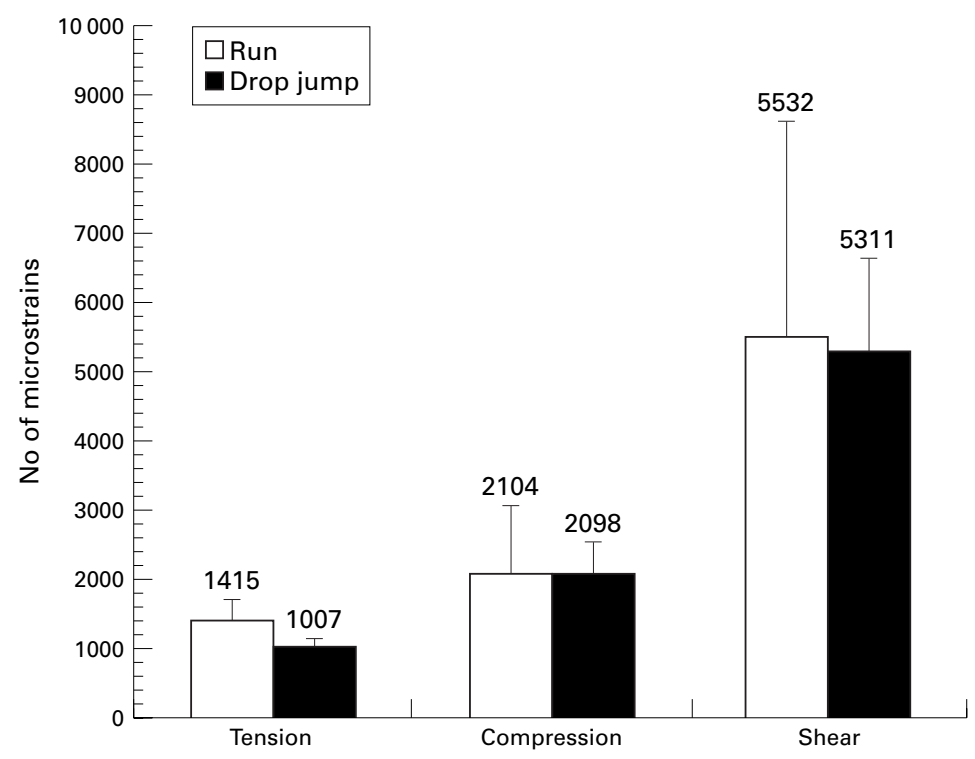

Figure 5 Mean principal tibial strains during running and drop jumping from $52 \mathrm{~cm}$. Error bars indicate SEM.

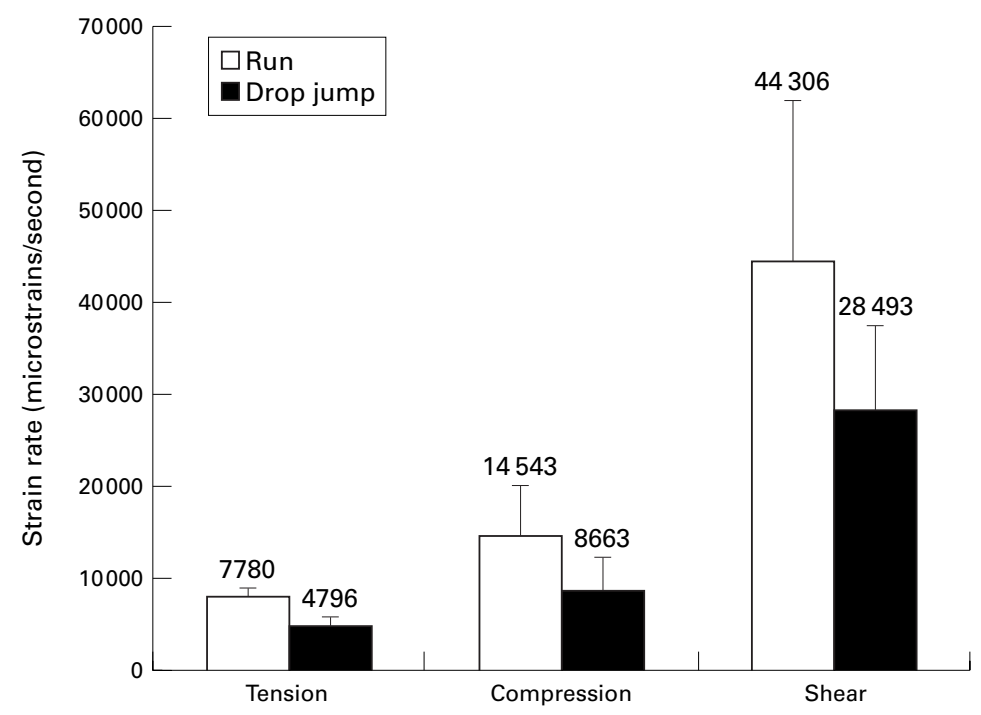

Figure 6 Mean principal tibial strain rates during running and drop jumping from 52 cm. Error bars indicate SEM. energy at each height was regressed against the maximum axial compression strains. Measurement of the range of angular motions of the ankle and knee joints for subjects at each jump height was achieved by idealising the lower extremities into stick figures based on picture sequences captured by a frame grabber from video jump recordings and displayed on Photoshop 5 (Adobe). The maximum principal strains and strain rates for $52 \mathrm{~cm}$ drop jumps were compared with those for running at 17 $\mathrm{km} / \mathrm{h}$ using paired $t$ tests.

\section{Results}

In four of the six subjects, all three strain gauged staples recorded for all three drop jumps, allowing calculation of maximum principal compression, tension and shear strains, and strain rates. Three of these subjects were men (aged 39, 45, and 52) and one a woman (33 years old). None of the subjects experienced tibial discomfort or pain while performing the jumps. At the time of surgical removal, all the staples were found to be firmly anchored in the bone. Only the four subjects with complete data sets were used for strain calculations. The principal strain and strain rates for the four subjects during walking before and after the run, and before and after the drop jumps varied by less than $10 \%$ (range $0.4-$ $9.6 \%)$.

There was no statistically significant difference in compression, tension, or shear strain with increasing drop jump heights (fig 1). There was a significant decrease $(p=0.03)$ in shear strain rate with increasing drop jump height (fig 2). At the $52 \mathrm{~cm}$ height, shear strain rate was reduced by one third.

There was no relation between maximum principal compression strain and calculated drop jump potential energy $\left(\epsilon_{\mathrm{c}}=1712.6+\right.$ $\left.1.00 \mathrm{E} ; r^{2}=0.05\right)$, indicating that subjects were able to dissipate part of the potential energy of successively higher drop jumps and not transmit it to their tibia (fig 3). This dissipation occurred through increased range of ankle and knee motion which tended to increase, although not significantly, with increasing drop jump height (fig 4). No statistically significant differences were found between the principal strains during running and drop jumping from $52 \mathrm{~cm}$ (fig 5), but compression $(\mathrm{p}=0.01)$ and tension $(\mathrm{p}=0.004)$ strain rates were significantly higher during running (fig 6).

The force plate reached saturation after the first drop jump height, and therefore ground reaction forces for higher drop jumps could not be calculated from force plate data.

\section{Discussion}

The physiology of landing has been studied in depth using force plates, video motion analysis, and accelerometry, in both the athletic and non-athletic populations. ${ }^{7-11}$ However, simultaneous in vivo bone strain measurements were not made in these previous studies.

Drop jumping was chosen to represent high impact exercises in this study. ${ }^{12}$ Although potential energy increases significantly with serially increased jump heights (fig 3), tibial strains and 
strain rates did not increase with increased jump height (figs 1 and 2). In fact, shear strain rates generated by jumps from 39 and $52 \mathrm{~cm}$ were only $70 \%$ of the shear strain magnitudes generated by drop jumps from $26 \mathrm{~cm}$. Likewise, compression and tensile strain rates at the greatest height were only about $65 \%$ of those at the lowest height, while shear strain rate at $52 \mathrm{~cm}$ was nearly half that at $26 \mathrm{~cm}$.

The failure to find that strains and strain rates increased, and in fact decreased in some instances, even in the presence of greater potential energy, shows that this additional energy was being dissipated in some fashion. This dissipation can be explained by the jumper's use of muscular and kinetic shock absorbing mechanisms. ${ }^{13}$ Video recordings showed that the landing strategies of subjects were similar. The range of knee and ankle motion during landing increased with increasing jump height. This was particularly true of motion at the ankle, which increased by $66 \%$ at greater heights. These findings parallel those of McNitt-Gray, ${ }^{11}$ who noted that, when skilled athletes land from jumps of increasing height, their knee flexion increases significantly. Dufek and $\mathrm{Bates}^{8}$ also showed that subjects modified their drop jump landing technique according to the height of the jump.

Previously, we showed that running produced tibial strains and strain rates two to three times that during walking. ${ }^{14}$ We hypothesised that high impact loading exercises would result in even higher strain and strain rates, and therefore place bone at increased risk for stress fracture. However, tibial strains during drop jumping from $52 \mathrm{~cm}$ were nearly identical with those during running at $17 \mathrm{~km} / \mathrm{h}$. Strain rates at the lower jump heights were also equivalent to those during running, but at the highest height $(52 \mathrm{~cm})$ were significantly lower than strain rates generated during running. Again, muscular and kinetic shock absorbing mechanisms during drop jumping can explain this lower strain rate. ${ }^{15}$ We suggest that the body uses these mechanisms to keep the strain levels and strain rates within normal physiological ranges to minimise damage to the bone. For example, Pattin et $a l^{16}$ showed that tensile strains below $2500 \mu \varepsilon$ and compressive strain below $400 \mu \varepsilon$ do not cause substantial damage to bone, but strains above these levels will accelerate fracture exponentially. Similar relations could be expected with strain rate. Pain was not a factor affecting jump technique in this experiment because none of the subjects experienced tibial discomfort or pain during the jumps.

It is likely that muscular shock absorbing mechanisms are most effective when a subject is not fatigued from overexercise. Yoshikawa et $a l^{17}$ showed that principal strains on the canine tibia increase by about $30 \%$ during a walking exercise when muscles become fatigued. The ability of muscles to respond quickly is impaired when they are fatigued, ${ }^{18}$ because the transfer of energy between concentric and eccentric phases of muscle contraction is slowed. ${ }^{19}$ In humans, this is associated with a $50 \%$ increase in tibial acceleration during normal gait, ${ }^{20}$ because the muscles are less capable of dissipating impact energy through eccentric contraction, ${ }^{131-23}$ and it is possible that their ability to reduce strains and strain rates under such conditions will be impaired. Therefore the results of this study apply only if the muscles are not fatigued.

There are several limitations to this study that should be considered when interpreting the results. Firstly, the strains were measured at only one specific site on the tibia and may not reflect other anatomical sites. Secondly, strains were measured using strain gauged bone staples placed in a $30^{\circ}$ rosette pattern rather than a rosette strain gauge directly bonded to the tibia. The latter technique was not used because it requires relatively invasive surgery for human experimentation. Measurement of bone strain is difficult to accomplish. Great care must be taken to insert the strain gauged staples exactly and to ensure that gauges are functioning properly during the tests. Although we took great care to analyse the recordings to ensure that the gauges were functioning properly, it is of course not possible in an in vivo experiment to test the gauges in the bone ex vivo after the experiment. Thirdly, the complexity of in vivo strain measurements, particularly in people, prevents these tests being accomplished on large numbers of subjects, and gauge failure can often occur. Therefore the sample size in this study is small. This reduces the likelihood that real changes will achieve the level of statistical significance. Fourthly, as noted above, the results apply only to conditions in which the subject is not fatigued and in whom muscular and joint shock absorbing mechanisms are intact. Fifthly, measurements of ground reaction forces generated in different planes on landing from different heights would have provided substantial additional information to allow us to interpret differences between and within subjects in landing strategy, and to compare these with the calculated energy of the jump and the amount of joint movement used to dissipate this energy. However, the force plate was saturated with jumps higher than $26 \mathrm{~cm}$, and so this was not possible. Finally, the effects of age and gender could not be considered in this study. Bassey et $a l^{4}$ found that an exercise programme based on repetitive jumping from a $8.5 \mathrm{~cm}$ height increased bone mineral density in premenopausal women but not in postmenopausal women. This study did not include postmenopausal subjects. There is some evidence that age may influence the magnitudes of strain and strain rate, as well as the strategy of dissipating energy by muscular and kinetic mechanisms. ${ }^{25}$ Gender may as well, although we are unaware of studies that have examined this. It was not a goal of this study to pursue these additional variables, but, given the association of strain rate and energy dissipation found in this study, in future work these variables could be incorporated into the study designs.

The original hypothesis of this study that high impact exercises result in higher tibial strains and strain rates than moderate impact loading exercises is not supported by the tibial strain gauge data. Exercises that generate high strain and strain rates increase risk for bone 
fatigue and stress fracture ${ }^{26}$ and should be avoided if not essential to training goals. This study indicates that, on the basis of their tibial strain and strain rates, high impact exercises are unlikely to increase risk for tibial fatigue in an athlete who is accustomed to fast running.

The research was supported by grant 3226 from the Office of the Chief Scientist, Ministry of Health, State of Israel and a grant from the National Operating Committee on Standards for Athletic Equipment.

Contributors: $C M$, the principal investigator, initiated the research, participated in study design, analysis and interpretation of the data, and writing of the paper, and was an experimental subject for the study. A F wrote the computerised program that calculated principal strains and strain rates from the raw data and was an experimental subject for the study. A S participated in study design and was responsible for the in vitro participat in testing and validation of the rosette pattern strain gauged staples. I E developed the strain gauged staple, performed in vitro and in vivo validation of the strain gaged staples, and was
an experimental subject. S M was responsible for photography, an experimental subject. $S M$ was responsible for photography, motion analysis, and was an experimental subject. $M \mathrm{M}$ was an
experimental subject and helped write the paper. $\mathrm{M}$ N was experimental subject and helped write the paper. $\mathrm{M} \mathrm{N}$ was
responsible for development of the electronics and was an responsible for development of the electronics and was an
experimental subject. N B participated in the study design and experimental subject. N B participated in the study design and
was responsible for data collection and quality control of the was responsible for data collection and quality control of the
experiment. D B participated in study design, was responsible for the interpretation of data, and edited the paper.

1 Burr DB. Muscle strength, bone mass, and age-related bone loss. F Bone Miner Res 1997;12:1547-51.

2 Frost HM. Why do marathon runners have less bone than weight lifters? A vital biomechanical view and explanation Bone 1997;20:183-9.

3 Burr DB. Bone, exercise and stress fracture. Exerc Sci Sport Rev 1997;25:171-94.

4 Milgrom C, Giladi M, Stein M, et al. Stress fracture in military recruits. A prospective study showing an unusually high incidence. f Bone foint Surg [Br] 1985;67:732-5.

5 Ekenman I, Halvorsen K, Westblad P, et al. Local bone deformations at two predominant sites for stress fractures
of the tibia: an in vivo study. Foot Ankle 1998;7:479-84. 6 Rolf C, Westbaid P, Ekenman I, et al. An experimental in vivo method for analysis of local deformation on tibia with simultaneous measures of ground reaction forces, lower
extremity muscle activity and joint motion. Scand $\mathcal{F}$ Med Sci extremity muscle activity
Sport 1996; 7:144-51.

7 Caster BL, Bates BT. The assessment of mechanical and neuromuscular response strategies during landing. Med Sci Sports Exerc 1995;27:736-44

8 Dufek JS, Bates BT. The evaluation and prediction of impact forces during landings. Med Sci Sports Exerc 1990;22:370-7
9 Gross TS, Nelson RC. The shock attenuation role of the ankle during landing from a vertical jump. Med Sci Sports ankle during landing from
Exerc 1998;20:506-14.

10 McKinley P, Pedotti A. Motor strategies in landing from a jump: the role of skill in task execution. Exp Brain Res 1992;90:427-40.

11 McNitt-Gray JL. Kinetics of the lower extremities during drop landings from three heights. F Biomech 1993;26:103746.

12 Jarvinen TL, Kannus P, Sievanen H, et al. Randomized controlled study of effects of sudden impact loading on rat trolled study of effects of sudden impact
femur. F Bone Miner Res 1999;13:1475-82.

13 McMahon T. Muscles, reflexes and locomotion. Princeton, NJ: University Press, 1984

14 Burr DB, Milgrom C, Fyhrie D, et al. In vivo measurement of human tibial strains during vigorous activity. Bone 1996; 18:405-10.

15 Wosk J, Voloshin AS. Wave attenuation in skeleton of young healthy persons. $\mathcal{F}$ Biomech $1981 ; 14: 261-7$.

16 Pattin, CA, Caler WE, Carter DR. Cyclic mechanical property degradation during fatigue loading of cortical bone. $\mathcal{F}$ Biomech 1996;29:69-79.

17 Yoshikawa T, Mori S, Santiesteban AJ, et al. The effects of muscle fatigue on bone strains. F Exp Biol 1994;188:21733.

18 Voloshin A, Mizrachi J, Verbitsky O, et al. Dynamic loading on the human musculoskeletal system: effect of fatigue. Clinical Biomechanics 1998;13:515-20.

19 Gollhofer A, Moki PV, Miyashita M, et al. Fatigue during stretch-shortening cycle exercise: changes in mechanical performance in human skeletal muscle. Int $\mathcal{F}$ Sports Med 1987;8:71-8.

20 Schultz AB, Ashton-Miller JA, Alexander NB. What leads to age and gender differences in balance maintenance and recovery? Muscle Nerve Supply 1997;5:60-4.

21 Hill DB. Production and absorption of work by muscle. Science 1962;131:897-903.

22 Paul LL, Munro PJ, Abernethy SR, et al. Musculoskeletal shock absorption: relative contribution of bone and soft tissues at various frequencies. $\mathcal{F}$ Biomech 1978;11:237-9.

23 Radin EL, Whittle MW, Yang YH, et al. The heelstrike transient, its relationship with the angular velocity of the shank and the effects of quadriceps paralysis. In: Lantz SA, King AI, eds. Advances in bioengineering. New York: The American Society of Musculoskeletal Engineers, 1986:121-3.

24 Bassey EJ, Rothwell WC, Littlewood JJ, et al. Pre- and postmenopausal women have different bone mineral responses to the same high-impact exercise. F Bone Miner Res 1998;13:1805-13.

25 Fyhrie DP, Milgrom C, Hoshaw SJ, et al. Effect of fatiguing exercise on longitudinal bone strains as related to stress fracture in humans. Annals of Biomechanical Engineering 1998;26:660-5.

26 Carter, DR, Caler, WE, Spengler, DM, et al. Fatigue behavior of adult cortical bone. The influence of mean strain and strain rate. Acta Orthop Scand 1981;52:481-90.

\section{Take home message}

High impact exercises, as represented by drop jumping in this experiment, do not produce higher tibial strains and strain rates than running and therefore are unlikely to place an athlete who is accustomed to fast running at higher risk for bone fatigue or stress fracture.

\section{Commentary}

Tibial stress fracture is a common injury in athletic and military populations. Bone strain arising from repetitive loading can lead to initiation and accumulation of microdamage. If this is not adequately repaired by the remodelling process, then a stress fracture can result. Our understanding of stress fracture development is hindered by the ethical and practical limitations associated with human studies. The authors are to be congratulated for obtaining in vivo tibial bone strain data during the activities of jumping and running. While the difficulties are acknowledged, including small sample and problems with strain gauge technology, the results of this study did not provide support for the concept that high impact loading in the form of drop jumping produces higher principal strains and strain rates than fast running. As the authors highlight, these results apply to the non-fatiguing exercise. The calf muscles in particular have a role in attenuating the load on the tibia. The authors conclude that hight impact exercise need not be avoided in training programmes. However, it may be the change in training rather than the absolute training that is the more important stimulus for stress fracture development. Thus high impact exercise may increase the risk of stress fracture if introduced suddenly into a training programme. Furthermore, the cause of stress fractures is likely to be multifactorial and training parameters will interact with other factors such as bone density and bone geometry.

KIM BENNELL Senior Lecturer, School of Physiotherapy, University of Melbourne PETER BRUKNER 